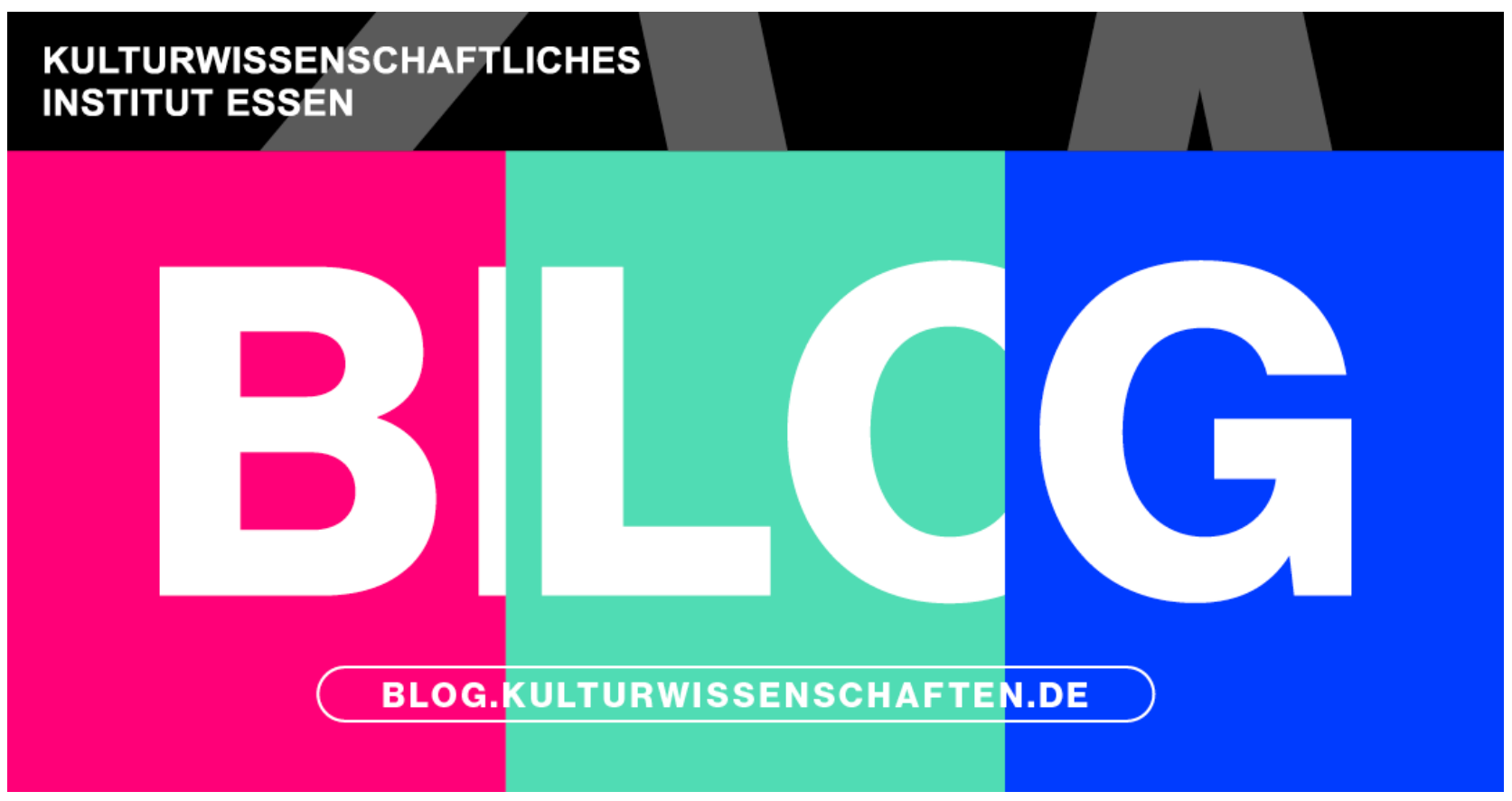

\title{
Unterhaltungsmagazine
}

\section{Zum Umgang der Experten des Boulevards mit COVID-19}

Erschienen in: Populäre Expertise | Popular Expertise Von: Maren Lickhardt

[Dieser Beitrag in der Reihe „Populäre Expertise" erscheint parallel auf dem KWI-Blog. und dem Blog_des SFB 1472 „Transformationen des Populären“.]

Wie gehen Boulevardmagazine mit Covid-19 um? Mit dieser Frage habe ich zwischen Februar und Juli 2020 sowie Oktober bis Dezember 2021 gefühlt so ziemlich alles durchgelesen, was man im Kiosk an illustrierten Magazinen finden kann. Insgesamt wurden ca. 120 Ausgaben eingesehen, darunter in unregelmäßigen Intervallen: Bild der Frau, Brigitte, Bunte, Closer, Cosmopolitan, Das goldene Blatt, Das neue Blatt, die aktuelle, Elle, Frau aktuell, Frau im Spiegel, Freizeit Revue, Freundin, Für Sie, Gala, Gala Style, Glamour,InTouch, Inside, In. Star News, InStyle, Jolie, MySelf, Neue Post, Neue Welt, neue woche, Tina, Vogue, Woche direkt. Ich habe jede Erwähnung der Pandemie registriert und kategorisiert. Insofern liegt eine ordentliche Datenbasis vor. Dieser Essay präsentiert allerdings keine quantitative Inhaltsanalyse, sondern reflektiert spezifische Lektüreeindrücke in einer bestimmten zeitlichen Dynamik.

Februar bis Juli 2020 
Nicht wenige illustrierte Unterhaltungsmagazine thematisieren Covid in den ersten Wochen und Monaten der Pandemie auf dem Cover. Hier trifft die Krise nicht nur auf Prominente, sondern vor allem auf: andere Krisen. Z.B. ist die ältere Generation der englischen Royals außer Gefecht gesetzt - Philipp und die Queen aus Altersgründen in Isolation, Charles erkrankt und in Quarantäne, von Andrew lieber ganz zu schweigen -, und vor diesem Hintergrund kann umso mehr herausgestellt werden, wie pflichtbewusst William und Kate tun, was Royals eben so tun, während - und das ist im Grunde das eigentliche Thema - Harry und Meghan desertiert sind. ${ }^{1}$ Über Boris Becker gibt es zu berichten, er „knutscht sich durch die Krise ${ }^{2}$, weil er in engerem Kontakt mit einer Frau gesichtet wurde. Indem der Körperkontakt mit Blick auf das Abstandsgebot kommentiert wird, disambiguiert der Artikel die Krise, denn bei Becker könnte es ja immer auch um andere Krisen gehen - Ex-Partnerinnen, Sorgerecht, Finanzen usw. Außerdem werden nun die Beziehungen diverser Stars hinsichtlich der Frage durchdacht, wer nun zusammenbleibt und wer sich trennt. ${ }^{3}$ Aber weil Boulevardmedien das immer tun, ändert sich nicht viel, sondern es wird nun die Prominenz zusätzlich mit dem Populären der Pandemie aufgeladen. Phatisch und metakommunikativ wird mitgeteilt, dass man in den Redaktionen versteht, was tagespolitisch ganz oben auf der Agenda steht, auch wenn man im Grunde nichts zum Thema beizutragen hat. Das zeigt sich auch an Formulierungen wie „Auch in Corona-Zeiten [...]"4, die zu Kochrezepten, Gymnastiktipps etc. überleiten. Man muss zeigen und sich wechselseitig bestätigen, dass man Covid im Blick hat, auch wenn die Selektionskriterien und die Themen der Boulevardberichterstattung konstant bleiben.

Die Zeitschriften als Experten für Konsum und Lifestyle, also z.B. Schminken und Frisieren, die nicht selten die Körperkultpraktiken mehr oder weniger Prominenter thematisieren, müssen es fast zwangsweise als worst case-Szenario ausweisen, wenn bestimmte Schauspieler:innen nicht mehr zum Friseur gehen können. Vor dem Hintergrund ist es vielleicht nicht verwunderlich, warum sich die Sorge in manchen Teilen der Bevölkerung schon früh von der Krankheit weg hin zu verlorenem Lebenswandel verschoben hat. Es ist in diesem Kontext bezeichnend, dass zwischen März und Mai 2020 sehr häufig pauschal von der negativ konnotierten ,Quarantäne‘ die Rede war einem Wort, das eigentlich eine mehr oder weniger medizinische Definition hat und die Isolation potentiell infektiöser Personen umschreibt -, auch wenn eigentlich fast immer nur das social distancing gemeint war. Diese Umsemantisierung dramatisiert einen vergleichsweise erträglichen Umstand und ignoriert die Gefahr des Virus, wobei der Isolation eine positive Auswirkung zugestanden wird: „Faulsein ist ausdrücklich erlaubt." ${ }^{5}$ Die eigentlichen Probleme im Zuge des Lockdowns, z.B. arbeitende Eltern zwischen Home-Office und Home-Schooling, hatten die meisten Zeitschriften bei aller Dramatisierung der Isolation nicht im Blick.

Es gibt auch Zeitschriften, die nicht versuchen, das Populäre mit dem Populären der Pandemie aufzupeppen. Dies ist dann der Fall, wenn sie eine eigene differenzierte Spezialisierung aufweisen, z.B. Mode. In der InTouch, die Mode neben vielem verhandelt, muss diese noch zur Pandemie passen. So gibt es Tipps für stylishe Gummistiefel und Regenmäntel, weil man ja noch spazieren gehen dürfe. ${ }^{6}$ Die deutlich auf Mode spezialisierten MySelf, InStyle, Gala Style, Cosmopolitan und Vogue dagegen bringen 
nichts oder so gut wie nichts zu Covid. Die Vogue nutzt noch nicht einmal offensichtliche Vorlagen, um ihre Themen an das populärste Thema des Jahres zu knüpfen. In einem Artikel zu Essen als Form der Gesellschaftskritik und den „Footprints“, die unsere Lebensstile hinterlassen, werden mögliche Zusammenhänge zwischen Fleischkonsum und Zoonosen ebensowenig thematisiert wie die vielleicht auch positiven ökologischen Folgen der Pandemie. ${ }^{7}$ In der Vogue Business werden Frauen in wichtigen und interessanten Berufen vorgestellt, und hier geht es z. B. um Anne Flörcken aus der Berliner Charité, ihres Zeichens Spezialistin für metastasierende Nierenzellenkarzinome. ${ }^{8}$ Fast jede andere Zeitschrift hätte vermutlich nach einer Virologin gesucht. Nicht so die Vogue, die sich aufgrund ihrer klaren Spezialisierung auf Mode- und Lifestyle-Themen nicht von der Pandemie irritieren lässt, die ihren Expertenstatus in den besagten Gebieten ausspielt und damit so über der Krise steht wie ein Chanel-Kostüm über den konkreten Kontext seines Einsatzes erhaben ist.

\section{Oktober bis Dezember 2021}

In dieser Phase der Pandemie, in der schon Routinen etabliert sind, zeigt sich insgesamt ein differenzierteres Bild als zu Beginn. Der Ton wird ernster, und es kommen viel häufiger Expert:innen aus verschiedenen Disziplinen zu Wort. Im Interview mit der Medizinethikerin Prof. Dr. med. Alena Buyx wird die Frage gestellt, ob die Pandemie unser Verständnis von Ethik geändert hat. ${ }^{9}$ Aber weil Ethik - außer vielleicht als Teilbereich von Philosophie und Theologie - keine Angelegenheit ist, für die es per se Expert:innen gibt, können sich die Magazine auch mit alltagsethischen Erwägungen einschalten, etwa indem konstatiert wird: „Die ich-zentrierten Zeiten sind vorbei, Freiheit ist ohne Verantwortung nichts wert." 10 Auch einer wütenden Krankenschwester wird das Wort erteilt, die über das politische Versagen beim Pandemiemanagement klagt, den Wahlkampf-Missbrauch des Freedom Day trotz unterbesetzter Kliniken kritisiert und betont, dass die vierte Welle niemanden hätte überraschen dürfen. ${ }^{11}$

Geradezu didaktisch im Sinne der dominierenden wissenschaftlichen und politischen Einschätzung versuchen die Magazine, die Impfung im Allgemeinen und den Booster im Speziellen unter die Leute zu bringen: „Jetzt boostern, Weihnachten sicher sein“. 12 Während Impfverweigerung als zu bereuender „Leichtsinn“ ausgewiesen wird, ${ }^{13}$ findet der Hashtag \#AllesindenArm sowie z. B. die Haltung der Schauspielerin Natalia Wörner, nur mit Geimpften zu arbeiten, affirmierend Erwähnung. ${ }^{14}$ Es wird auch darauf verwiesen, dass viele andere Maßnahmen zurückgefahren werden könnten, wenn wir eine höhere Impfquote hätten. ${ }^{15}$ Informationen zu Impfungen - auch zu neuen Impfstoffen - werden nicht selten explizit von medizinischen Expert:innen bezogen. Der Virologe Dr. Jan Leidel wird allgemein zu Impfungen befragt, ${ }^{16}$ Booster-Empfehlungen der STIKO mitgeteilt ${ }^{17}$ - die gleichwohl kurz nach Erscheinen der Zeitschriften teilweise schon veraltet waren -, Berechnungen der HU Berlin zur geringeren Ansteckungsgefahr durch Geimpfte werden präsentiert, ${ }^{18}$ Prof. Hubert Wirtz von der Post-Covid-Ambulanz in Leipzig klärt über Long Covid auf, ${ }^{19}$ Prof. Gernot Marx erklärt, was es mit Impfdurchbrüchen auf sich hat. ${ }^{20}$ Usw. usf. Sehr bekannte, populär gewordene Namen finden sich hier nicht, aber durch die Nennung des Titels, der genauen Ausrichtung der Tätigkeit sowie der Wirkstätte der Personen wird der Expert:innen-Status bezeugt. Wo die 
Expert:innen zu Wort kommen, wird auch auf die interne Differenzierung und Spezialisierung der Zeitschriften gesetzt, d.h. zumeist befinden sich die Informationen in den jeweiligen Gesundheitsratgeberressorts der Magazine.

Frau im Spiegel, neue woche und tina scheinen in ihren Impfkampagnen mit ihrer Wortwahl auf Feigheit oder Kindlichkeit von Impfverweigernden anzuspielen, indem die Impfung als „Piks“ bezeichnet wird. ${ }^{21}$ Die Neue Post unterstell direkter eine Angststörung, wenn sie darauf verweist: „Gegen Spritzen-Phobie kann man etwas tun." 22 Insgesamt findet in dem Segment eine klare Positionierung pro Impfung statt. Zugleich ist man bemüht, Impfgegner:innen nicht durch allzu harte Kritik zu verprellen. So schöpfen die Zeitschriften ihre Expertise in Sachen positive Leser:innenansprache aus und wahren dabei die Balance zwischen tantenhafter, freundschaftlicher Ansprache auf Augenhöhe und dem Zitieren medizinischer Expert:innen.

Aber nicht nur sind die Zeitschriften im weiteren Verlauf der Pandemie insgesamt bemüht, Expert:innen sprechen zu lassen, sondern sie thematisieren auch Pseudo-Expertisen. So heißt es über Michael Wendler, er verbreite Fake News zum Tod von Mirco Nontschew, indem er diesen mit Impfungen in Verbindung bringt, was „leider" keine rechtlichen Konsequenzen für Wendler habe. ${ }^{23}$ An anderer Stelle wird Wendler als „Verschwörungsschwurbler" bezeichnet. ${ }^{24}$

Insgesamt taucht das Thema Covid auch unabhängig von Impfungen jetzt weit häufiger in Bezug auf medizinische Fragen auf als in der ersten Phase, weil sich ein gewisser Wissensstand konsolidiert hat, der nun auch im Wochen- und Monatsrhythmus popularisiert werden kann. Dabei reicht das Spektrum vom individuellen wie gesetzlich verordneten Schutz vor der der Krankheit selbst ${ }^{25}$ über Mutationen ${ }^{26}$ - wobei Omikron die Zeitschriften schnell veraltet wirken lässt - bis zu den unerwünschten Nebenwirkungen der Maßnahmen wie unreine Haut durch das Maskentragen ${ }^{27}$ oder Depression durch Isolation ${ }^{28}$. Teilweise werden die Themen, wie bereits gesagt, in den medizinischen Ressorts ausgetragen; teilweise passen sie in die allgemeineren Beautyund Lifestyle-Seiten.

Das Themenspektrum rund um Covid ist im Boulevardjournalismus breiter geworden. Die Breite resultiert allerdings nicht daraus, dass jedes boulevardeske Thema allein assoziativ aufgeladen wird, wie dies in der ersten Phase oft der Fall war. Vielmehr sind nach eineinhalb Jahren alle Lebensbereiche kausal von der Pandemie betroffen. Stricken kann man nun nicht auch in der Pandemie, sondern die Pandemie hat es aufgezwungen, häusliche Hobbys zu entdecken. ${ }^{29}$ Kochrezepte sind nicht auch in der Pandemie nach wie vor hilfreich, sondern die Pandemie hat aufgrund geschlossener Restaurants neue Herausforderungen hinsichtlich des Abwechslungsreichtums mit sich gebracht oder überhaupt die Einstellung zum Kochen verändert. ${ }^{30}$ Zudem wird reflektiert, dass Themen wie häusliche Gewalt, Überforderung, Langeweile, Nähe- und/oder Abgrenzungsbedürfnisse durch Covid eine neue Dimension erhalten haben. ${ }^{31}$

Überlegungen 
Es ist auffällig, dass in der zweiten Screening-Phase viel häufiger Expert:innen Pandemie-bezogener Disziplinen das Wort überlassen wird als in der ersten. Da in der Zwischenzeit sehr viel über das Verhältnis von Laienmeinungen und Expert:innenwissen debattiert wurde, ist nicht auszuschließen, dass hier ein bewusster Lernprozess stattgefunden hat.

Unterhaltungsmagazine brauchen Routinen, müssen aber gleichzeitig halbwegs aktuell sein. Sie können erst dann Expert:innenwissen popularisieren, wenn dieses konsolidiert ist. Dann greift auch die interne Differenzierung der Blätter, und die Gesundheitsratgeber können spezifisch genutzt werden. Solange dies nicht möglich ist, muss auf diffuse und unspezifische Weise die Popularität der Pandemie selbst zum Thema gemacht werden. Das heißt, es stellt sich zunächst eine quasi-phatische Kommunikation ein, die mitteilt, dass man weiß, woran Leser:innen denken. Sie setzen zu Beginn den Akzent auf ihre Kernkompetenz Lifestyle oder verhinderter Lifestyle. Dabei schöpfen sie die ersten Reflexe auf die Pandemie ab und fungieren möglicherweise als deren Verstärker.

Abgesehen von Modezeitschriften bildet die ausgewogene, forciert korrekte und ,komplexe‘ Brigitte eine Ausnahme. Sie eröffnet von Beginn an ein großes Spektrum an Pandemieproblemen insbesondere für Frauen und stellt diverse weibliche Leistungen zu deren Bewältigung heraus. Dabei ist sie stets bemüht, das aktuelle Thema ,sinnvoll‘ an Themen zu knüpfen, die eher eine strukturelle statt einer punktuellen Dimension aufweisen; hier geht es nicht nur um Konsum, sondern um Herausforderungen im Alltag, Vereinbarkeit von Familie und Beruf, Wohltätigkeit und soziales Engagement von Frauen, Bewältigung von Lebenskrisen, starke weibliche Rollenvorbilder. Auch in der Pandemie unterscheidet sich die Brigitte von anderen Zeitschriften dadurch, dass sie eher auf langfristige, gleichbleibend aktuelle Themen setzt und nicht so stark akute Aktualität kommuniziert.

Sobald die Zeitschriften ihren ersten Reflex, Laienmeinungen Prominenter zu thematisieren, überwunden haben, steht in diesen in keiner Weise in Frage, dass Mediziner:innen, Naturwissenschaftler:innen und Jurist:innen auf ihren jeweiligen Gebieten als maßgebliche Instanz anzuerkennen sind. Die Zeitschriften können ein eigenes - diffuses - Skript liefern, wenn es darum geht, aktuelle Stimmungsbilder zu reflektieren oder zu erzeugen; sie setzen aber seriös auf das Skript der Expert:innen, sobald dieses ansatzweise zur Verfügung steht. Insofern ist hier eine deutlich geringere selbstreferentielle Dynamik populärer Empfindungen zu verzeichnen als in Blasen sozialer Medien.

\section{References}

1. Z.B. Gala, Apr.; In Touch, Apr.; Frau im Spiegel, Apr.

2. die aktuelle, Mrz.

3. InTouch, Apr.; Bunte Mai.

4. Closer, 1/Mai, S. 13.

5. In Touch, Apr. 
6. InTouch, Apr.

7. Vogue, Apr.

8. Vogue, Apr.

9. Elle, Ausg. Jan. 22 (erscheint im Dez.), 67.

10. Bild der Frau, Nr. 51, Dez., Editorial.

11. Bild der Frau, Nr. 45, Nov., 5.

12. tina, Nr. 51, Dez., 50.

13. Neue woche, Nr. 48, Nov., 64.

14. Frau im Spiegel, Nr. 48, Nov., 45, 25.

15. Freizeit Revue, Nr. 45, Nov., 74.

16. Freundin, Nr. 28, Nov., 106.

17. tina, Nr. 45, Nov., 55.

18. tina, Nr. 51, Dez., 50.

19. tina, Nr. 51, Dez., 50.

20. tina, Nr. 45, Nov., 55.

21. Frau im Spiegel, Nr. 52, Nov., 25; tina, Nr. 51, Dez., 50; neue woche, Nr. 51, Dez., 62.

22. Neue Post, Nr. 48, Nov., 58.

23. InTouch, Nr. 51, Dez., 14.

24. Closer, Nr. 51, Dez., 20.

25. neue woche, Nr. 51, Dez., 49; Das goldene Blatt, Nr. 50, Dez., 23; Freizeit Revue, Nr. 48, Nov., 71; Das neue Blatt, Nr. 48, Nov., 54; Bunte, Nr. 40, Sep., 85; tina, Nr. 45, Nov., 56.

26. Frau im Spiegel, Nr., Nov., 52; tina, Nr. 45, Nov., 57.

27. Das goldene Blatt, Nr. 50, Dez., 23.

28. Für Sie, Nr. 21, Sep., 44.

29. Jolie, Dezemberheft, 11.

30. Freundin, Nr. 24, Sep., 64, 98.

31. Bild der Frau, Nr. 45, Nov., 30; Brigitte, Nr. 23, Okt., 174.

SUGGESTED CITATION: Lickhardt, Maren: Unterhaltungsmagazine. Zum Umgang der Experten des Boulevards mit COVID-19, in: KWI-BLOG, [https://blog.kulturwissenschaften.de/unterhaltungsmagazine/], 31.01 .2022

DOI: https://doi.org/10.37189/kwi-blog/20220131-0830 


\section{DuEPublico}

Duisburg-Essen Publications online

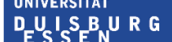

offen im Denken

Dieser Text wird via DuEPublico, dem Dokumenten- und Publikationsserver der Universität Duisburg-Essen, zur Verfügung gestellt. Die hier veröffentlichte Version der E-Publikation kann von einer eventuell ebenfalls veröffentlichten Verlagsversion abweichen.

DOI: $\quad$ 10.37189/kwi-blog/20220131-0830

URN: urn:nbn:de:hbz:464-20220131-111054-5

Alle Rechte vorbehalten. 